\title{
Artivity: Documenting Digital Art Practice
}

\author{
Athanasios Velios \\ University of the Arts London \\ Chelsea College of Arts \\ 16 John Islip Street \\ London SW1P 4JU \\ UK \\ a.velios@arts.ac.uk
}

\author{
Sebastian Faubel \\ Semiodesk $\mathrm{GmbH}$ \\ Werner-von-Siemens Str. 6 \\ Building 15K \\ D-86159 Augsburg \\ Germany \\ sebastian@semiodesk.com
}

\author{
Moritz Eberl \\ Semiodesk $\mathrm{GmbH}$ \\ Werner-von-Siemens Str. 6 \\ Building 15K \\ D-86159 Augsburg \\ Germany \\ moritz@semiodesk.com
}

\section{INTRODUCTION}

Arguments and interpretations made while researching the arts are based on historical evidence. The technical analysis of artworks and artists' archives supply such evidence with the objective being to provide answers on how and why an artwork was made, i.e. how can artistic practice be described? Identifying such evidence in digital art is difficult due to the lack of the artwork's physical manifestation. Available resources about the artwork often concern long-term digital preservation, e.g., Serexhe (2012), and not the practice. Information on the process and context of the artwork is typically lost. In this paper we present a method for capturing part of this contextual data. We also present an implementation in the form of a software tool called Artivity. We highlight a case study with data by the artist Gino Ballantyne. We discuss the limitations of our approach and the expected use of the tool.

\section{PREVIOUS WORK}

Recording user interaction can be done by tracking the use of peripheral devices (e.g. mouse tracking and keyboard logging). For example, Morgan at al. (2013) have reported on that. It can also be done by tracking the use of software commands. For example this was shown by Alexander, Cockburn \& Lobb (2008). The latter option allows easier reconstruction of the interaction in terms of the process used to reach a result, although the meaning of that interaction is again lost.

\subsection{Ontologies}

The NEPOMUK project (Groza 2007) showed how user interaction can be formalised semantically. It delivered a set of formal ontologies to cover desktop events, like opening files or visiting webpages. Implementations on the GNOME and $\mathrm{KDE}$ desktops soon followed. More recently the
World Wide Web Consortium recommended the PROV ontology (Lebo et al. 2013) for the description of provenance information. The production of a digital file (e.g. an artwork) can be considered as a set of activities modifying computer entities (e.g. image layers). By modelling these activities, the provenance of the file is recorded alongside the development of the artwork itself. We adopt this method for documenting user interaction and through our implementation with Artivity, we extend it to cover creative software.

\section{SOFTWARE ARCHITECTURE}

Artivity runs solely on the desktop as a background service and without any requirement for user interaction - therefore it does not interfere with practice. The software architecture is summarised in figure 1. Artivity collects data delivered by several software plugins for a) vector and raster image editing and b) web browsing. This is then stored in a database. Artivity Explorer is a separate application which connects to the service to allow querying the database. Artivity can be installed on MacOSX, Windows and GNU/Linux. Installation files for MacOSX and Windows can be downloaded from the software website (http://artivity.io).

\section{PRELIMINARY RESULTS}

For our early testing, we asked the artist Gino Ballantyne to work for a period of a few days with Artivity activated. One of the outputs can be seen in figure 2 while there were at least 40 relevant files present on the disk. Our target was to offer an interpretation on how and why this artwork was created. Given that each action is time-stamped Artivity data allowed us to map the interaction and order of the files produced. We could produce a visual slide-show of the development of the different components of the artwork and then show how they were combined in the final document. We 
could also collect information about the filters and tools used on a step-by-step basis.

To offer an interpretation on why the artwork was made, we queried the web browsing history. Two important resources came up. One about medieval manuscripts indicating that the thick lines could signify symbols. Another was an empty music score, visible at the background of the artwork, indicating that the symbols in the front could correspond to sound. Given that the artist was working in the studio of an art college while students were preparing for a show, an interpretation of the work would be that it captures the noise in the studio during the surge of activity for the preparation of the show.

\section{DISCUSSION AND FURTHER WORK}

The information recorded by Artivity is enough to offer a reasonable description of how a digital artwork was made. Further testing and development is required to provide provenance links between files when copy/paste operations from one file to another occurs. We have also undertaken some basic statistical analysis of the data. We have some promising results which can indicate the confidence of the artist when using the creative software and the speed with which the artist works. Further analysis is required and we will report our findings in the future.

\subsection{Supported Tools}

We are committed to open-source software but most our users work with proprietary software. We have delivered Artivity plugins for Adobe Illustrator and Adobe Photoshop as well as Inkscape, Krita, Firefox and Chrome. The platform can support additional plugins which can be written if there is demand.

\subsection{Software Adoption}

The software has been used by a handful of artists and although we are happy with its stability we are actively seeking to enlarge the community of users. We are targeting educational environments where we believe Artivity can provide an insight in the process of learning to use creative software. We have also noted that artists are interested in the the data Artivity captures about their practice and may consider Artivity as part of their toolbox.

\section{ACKNOWLEDGEMENTS}

The authors thank the artists who have contributed to Artivity so far and especially Gino Ballantyne. This project was funded and supported by JISC.

\section{REFERENCES}

Alexander, J., Cockburn, A. and Lobb, R. (2008) AppMonitor: A tool for recording user actions in unmodified Windows applications, Behavior Research Methods, 40(2), pp. 413-421.

Groza, T., H, S., Möller, K., Minack, E., Jazayeri, M., Mesnage, C., Reif, G. and Gudjónsdóttir, R. (2007) The NEPOMUK project - on the way to the social semantic desktop, Proceedings of 1 Semantics' 07, JUCS, pp. 201-211.

Lebo, T., Sahoo, S. and McGuinness, D. (eds) (2013) PROV-O: The PROV Ontology, W3C. https://www.w3.org/TR/prov-o/ (retrieved 17 March 2017).

Morgan, J. H., Cheng, C.-Y., Pike, C. and Ritter, F. E. (2013) A Design, Tests and Considerations for Improving Keystroke and Mouse Loggers, Interacting with Computers, 25(3), pp. 242-258.

Serexhe, B. (2012) Preservation of Digital Art: Theory and Practice. The digital art conservation project. Ambra Verlag, Vienna.

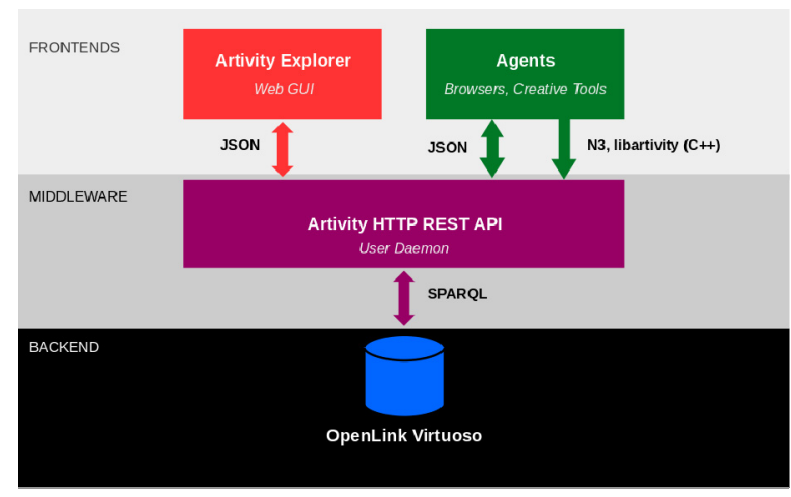

Figure 1: Artivity software stack.

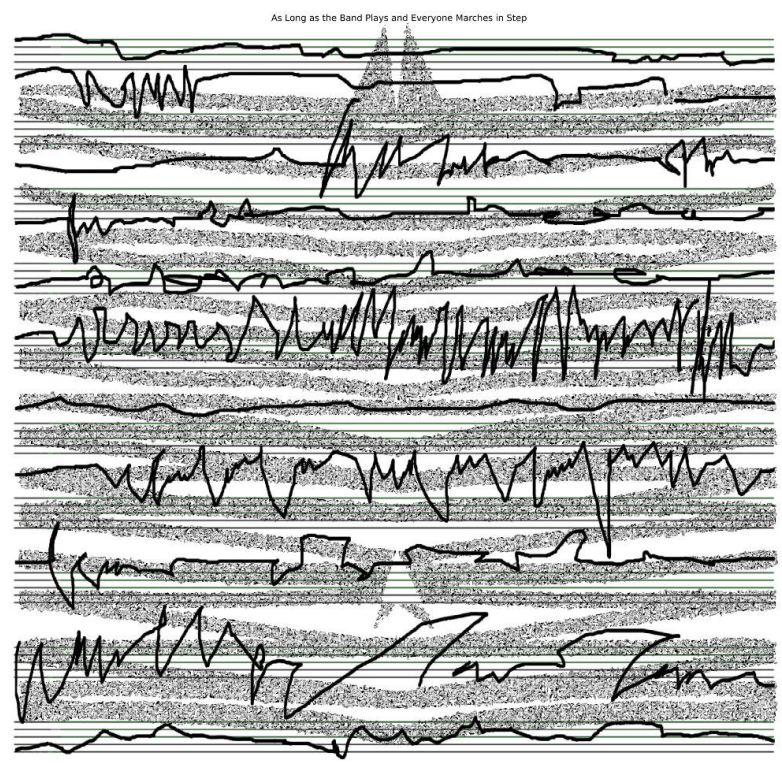

Figure 2: "As long as the band plays and everyone marches in step" by Gino Ballantyne. 